\title{
Older patients with differentiated thyroid cancer exhibit more aggressive pathological characteristics than younger patients
}

\author{
Jasper Johar, MD \\ Heidi Britton, BSc (Hons) \\ Sam M. Wiseman, MD \\ Accepted July 11, 2019 \\ Correspondence to: \\ S.M. Wiseman \\ Department of Surgery \\ St. Paul's Hospital \& University of British \\ Columbia \\ Rm C303, 1081 Burrard St \\ Vancouver BC V6Z 1Y6 \\ smwiseman@providencehealth.bc.ca
}

DOI: $10.1503 /$ cjs.017918

\section{SUMmarY}

Differentiated thyroid cancer (DTC) has long been recognized as having a worse prognosis in older people. We retrospectively evaluated the clinical and pathological characteristics of 973 sequentially treated patients with primary DTC stratified into 2 age groups, $\geq 55$ or $<55$ years, based on the current American Joint Committee on Cancer (AJCC) DTC staging system. We found that older patients had a higher frequency of extrathyroidal cancer extension and larger cancers, and that their cancers were less commonly completely resectable.

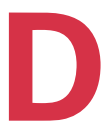
ifferentiated thyroid cancer (DTC) accounts for more than $95 \%$ of all newly diagnosed thyroid cancer cases in Canada and includes papillary (PTC) and follicular (FTC) histopathological subtypes. ${ }^{1}$ The incidence of DTC has been rising steadily for more than 3 decades in Canada and most other countries. ${ }^{1}$ In addition, for reasons that have remained poorly defined, DTC has been widely established as having a worse prognosis in older people. ${ }^{2}$ Current thyroid cancer prognostication systems, such as the Mayo Clinic's MACIS (metastases, age, completeness of resection, invasion, and size) score and the American Joint Committee on Cancer (AJCC) DTC staging system, that are used for surgical and adjuvant therapy decision making incorporate patient age as part of their risk stratification algorithms. ${ }^{2,3}$ We sought to determine if there were any statistically significant differences in the pathological characteristics of DTCs that were diagnosed in younger $(<55$ yr) compared with older ( $\geq 55 \mathrm{yr}$ ) patients.

We retrospectively reviewed 973 sequential primary DTC cases that were treated at St Paul's Hospital in Vancouver, Canada, between January 2001 and April 2017. At our institution, thyroid care is managed in accordance with the American Thyroid Association (ATA) guidelines for the management of adult patients with thyroid nodules and differentiated thyroid cancer. ${ }^{4}$

Thyroid microcarcinomas (DTC $<1 \mathrm{~cm}$ in diameter) as well as non-DTC thyroid cancer histological types, were excluded from the primary analysis. We retrospectively collected the following clinicopathological characteristics of DTC and evaluated their relationship with patient age: cancer size, focality, presence of extrathyroidal extension (ETE), presence of vascular invasion and completeness of cancer resection, patient sex, and the presence and extent of nodal or distant metastases. For the purpose of our analysis, ETE was defined as the presence of microscopic and/or gross ETE.

Of the 603 patients who made up the final study population after exclusion of 307 microcarcinoma cases and 63 non-DTC cases, 559 patients were diagnosed with PTC and 44 patients were diagnosed with FTC. Patient age was normally distributed (median 45, interquartile range 35-56). The mean age was 45.5 years in women $(n=452)$, and 48.0 years in men $(n=151)$.

We compared patient age using the recently revised age cut-point from the 8th edition of the AJCC DTC staging system $(55 \mathrm{yr})$ in order to determine whether there were statistical differences in the clinical and/or pathological 


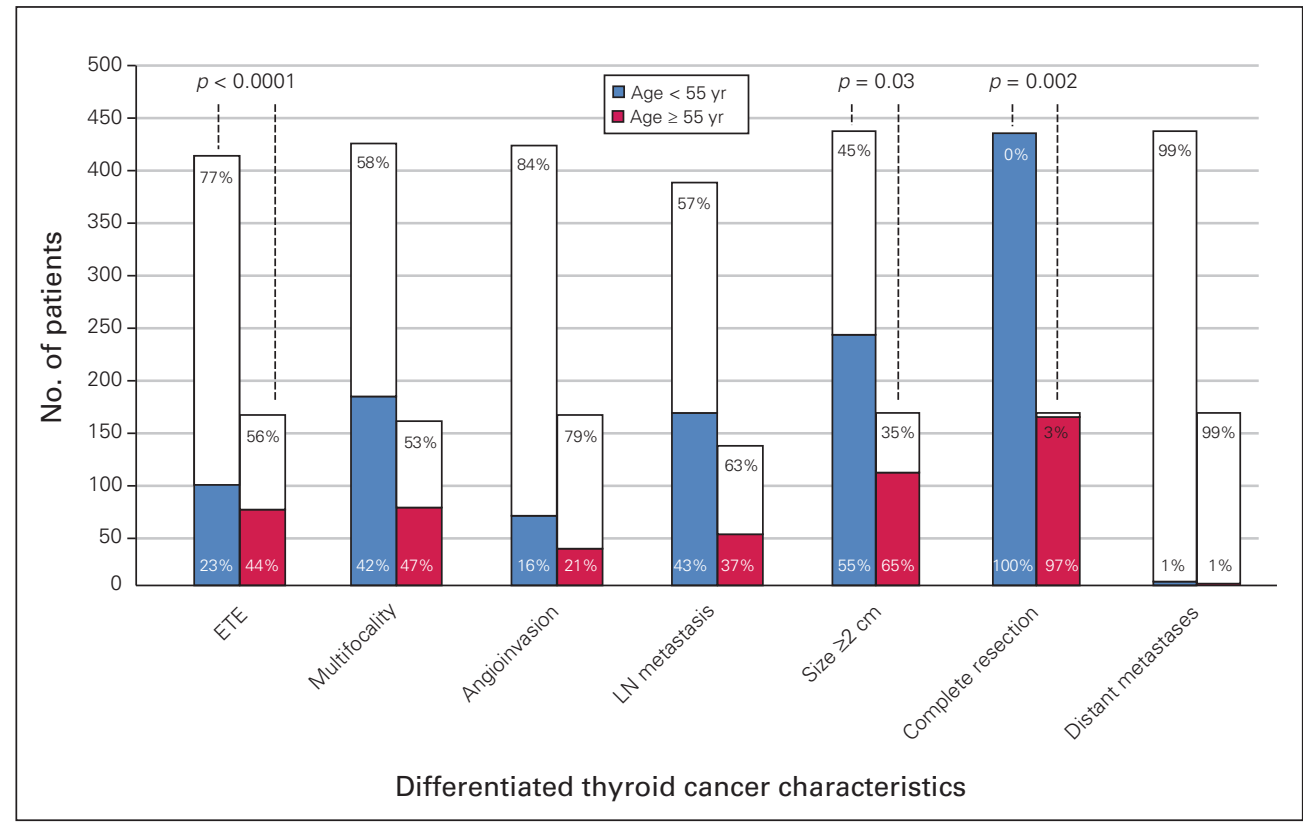

Fig. 1. Differentiated thyroid cancer characteristics compared between younger $(<55 \mathrm{yr}$ ) and older ( $\geq 55 \mathrm{yr}$ ) patients. ETE = extrathyroidal extension; $L N=$ lymph node; mets = metastases. The coloured portions of the bars represent the presence of the characteristics, whereas the white portions represent the absence of the characteristics. worse prognosis in older patients. Prior research suggests that more aggressive pathological characteristics are associated with worse survival in older patients with DTC when using the 7th edition AJCC thyroid cancer staging system cut-point of 45 years. ${ }^{5}$ These findings hold true, and are even more striking when using a cut-point of 55 years. Knowing that older patients exhibit more aggressive pathological findings may help surgeons with preoperative planning and patient counselling, ultimately allowing for more accurately tailored treatment of people with this increasingly common endocrine cancer. characteristics of older versus younger patients that contribute to the less favourable prognosis associated with DTC in older patients. A cut-point of $2 \mathrm{~cm}$ was used to compare size between the younger and older groups in order to determine the proportion of T1 compared with $\mathrm{T} 2$ and larger tumours in accordance with the 8th edition of the AJCC DTC staging system.

Younger patients differed significantly from older patients for ETE $(p<0.0001)$, cancer size $\geq 2 \mathrm{~cm}(p=0.003)$ and completeness of cancer resection $(p<0.0001)$, but not for multifocality, angioinvasion, or lymph node or distant metastasis (Fig. 1). When a cut-point of 45 years was evaluated, only ETE was found to be significantly different between the age groups. Although we were unable to distinguish the patients who had microscopic ETE from those who had gross ETE by pathology report review alone, the 5 patients with DTC that could not be completely resected all exhibited gross ETE, and they all were older than 55 years.

\section{Conclusion}

The cancers of older patients with DTC exhibit more aggressive pathological characteristics than those of younger patients; not surprisingly, they are less commonly completely resectable, which likely contributes to the
Acknowledgements: The authors thank Ms. Kaidi Liu, St. Paul's Hospital Department of Surgery research associate, for her administrative assistance that facilitated the conduct of this study.

Affiliations: From the Department of Surgery St. Paul's Hospital \& University of British Columbia, Vancouver, BC.

Competing interests: None declared.

Contributors: All authors contributed substantially to the conception, writing, and revision of this article and approved the final version for publication.

\section{References}

1. Vaccarella S, Franceschi S, Bray F, et al. Worldwide thyroid-cancer epidemic? The increasing impact of overdiagnosis. $N$ Engl 7 Med 2016;375:614-7.

2. Kazaure HS, Roman SA, Sosa JA. The impact of age on thyroid cancer staging. Curr Opin Endocrinol Diabetes Obes 2018;25:330-4.

3. Hay ID, Bergstralh EJ, Goellner JR, et al. Predicting outcome in papillary thyroid carcinoma: development of a reliable prognostic scoring system in a cohort of 1779 patients surgically treated at one institution during 1940 through 1989. Surgery 1993;114:1050-7.

4. Haugen BR, Alexander EK, Bible KC, et al. 2015 American Thyroid Association management guidelines for adult patients with thyroid nodules and differentiated thyroid cancer. Thyroid 2015;26:1-133.

5. Nixon IJ, Wang LY, Palmer FL, et al. The impact of nodal status on outcome in older patients with papillary thyroid cancer. Surgery 2014;156:137-46. 